\title{
Antiphospholipid antibodies associated with alcoholic liver disease specifically recognise oxidised phospholipids
}

R Rolla, D Vay, E Mottaran, M Parodi, M Vidali, M Sartori, C Rigamonti, G Bellomo, E Albano

\begin{abstract}
Background-Circulating antiphospholipid antibodies (aPL) are often detected in patients with alcoholic liver disease (ALD) but little is known about the causes of their formation.

Aims-We have evaluated whether ethanol mediated oxidative injury might promote the development of aPL in ALD.

Patients and methods-IgG against $\beta_{2}$ glycoprotein 1 ( $\beta_{2}-$ GP1), cardiolipin, and human serum albumin (HSA) complexed with either oxidised arachidonic acid (HSA-APP) or malondialdehyde (HSAMDA) were assayed by ELISA in heavy drinkers with or without ALD and in healthy subjects.
\end{abstract}

Results-Circulating IgG recognising cardiolipin were significantly higher in ALD patients than in controls. However, anticardiolipin reactivity of ALD sera was only evident using, as the antigen, oxidised cardiolipin but not oxidation protected cardiolipin. In ALD patients, individual values of IgG antioxidised cardiolipin were associated with the titres of antibodies against HSA-MDA and HSAAPP $(r=0.68$ and 0.72 , respectively; $\mathrm{p}<0.0001$ ) used as markers of oxidative stress. ALD patients also displayed increased levels of antibodies against phospholipid binding protein $\beta_{2}-$ GP1, and individual reactivity towards oxidised cardiolipin and $\beta_{2}-$ GP1 were highly correlated $(r=0.85 ; \mathrm{p}<0.0001)$. IgG binding to oxidised cardiolipin, HSA-MDA, and HSA-APP was also significantly higher in $\beta_{2}-$ GP1 positive than in $\beta_{2}-$ GP1 negative sera. However, preadsorption of $\beta_{2}-$ GP1 positive sera on $\beta_{2}-$ GP1 coated ELISA plates reduced reactivity to oxidised cardiolipin by $80 \%$, without affecting that to HSA-APP or HSA-MDA.

Conclusions-Ethanol induced oxidative injury is associated with the development of antibodies targeting complexes between oxidised cardiolipin and $\beta_{2}-$ GP1. These antibodies might account for high aPL titres observed in patients with severe ALD. (Gut 2001;49:852-859)

Keywords: oxidative stress; lipid peroxidation; $\beta_{2}$ glycoprotein 1; ethanol; autoantibodies

Antiphospholipid antibodies (aPL) belong to a heterogeneous group of autoantibodies with apparent specificity for negatively charged phospholipids. ${ }^{12}$ The presence of these antibodies characterises primary antiphospholipid syndrome, or Hughes' syndrome, consisting of recurrent vascular thrombosis, fetal losses, and thrombocytopenia. ${ }^{3}$ aPL and antiphospholipid syndrome are often associated with rheumatic and autoimmune diseases, such as systemic lupus erythematosus, rheumatoid arthritis, and systemic sclerosis. ${ }^{1-4}$ However, aPL are also present in patients with chronic infections (syphilis, malaria, human immunodeficiency virus 1), lymphoproliferative diseases, and sickle cell anaemia. ${ }^{1-4}$

The molecular targets of aPL have not yet been characterised in detail. aPL are usually identified by their ability to react with solid phase bound cardiolipin but other anionic phospholipids - that is, phosphatidylserine and phosphatidylethanolamine-can be recognised also. ${ }^{12}$ Studies by McNeil and colleagues ${ }^{5}$ and Galli and colleagues ${ }^{6}$ have shown that aPL binding to phospholipids is largely mediated by $\beta_{2}$ glycoprotein $1\left(\beta_{2}-\mathrm{GP} 1\right)$ or apolipoprotein $\mathrm{H}$, a plasmatic $50 \mathrm{kDa}$ glycoprotein which readily binds to phospholipids as well as to other negatively charged substances. ${ }^{4}$ Conformational changes occurring in $\beta_{2}$-GP1 following interaction with phospholipids render this protein antigenic to aPL. ${ }^{7}$ Indeed, $\beta_{2}$-GP1 can be recognised by aPL also in the absence of phospholipids when attached to irradiated polystyrene or nitrocellulose membranes that are rich in negative charges. ${ }^{8}$ None the less, phospholipid complexes with prothrombin, protein $\mathrm{C}$, and protein $\mathrm{S}$ can also be targets of aPL and this may contribute to the anticoagulant properties ascribed to aPL." Recently, Hörkkö et al have reported that epitopes originating during cardiolipin oxidation are recognised by aPL from women with primary antiphospholipid syndrome. ${ }^{9}$ They also suggested that the antigenic determinants might result from the reaction of $\beta_{2}-\mathrm{GP} 1$ with products derived from the peroxidative breakdown of cardiolipin unsaturated fatty acids. ${ }^{9} 10$

Recent studies have shown that aPL can be detected in patients with chronic liver disease,

Abbreviations used in this paper: ALD, alcoholic liver disease; aPL, antiphospholipid antibodies; $\beta_{2}$-GP1, $\beta_{2}$ glycoprotein 1 ; DPPD, diphenylphenylendiamine; $\mathrm{HCV}$, hepatitis $\mathrm{C}$ virus; HSA, human serum albumin; HSA-MDA, malondialdehyde adducts with human serum albumin; HSA-APP, adducts between human serum albumin and arachidonic acid oxidation products; HSA-LPP, adducts between human serum albumin and linoleic acid oxidation products; MDA, malondialdehyde; od, optical density; PBS, phosphate buffered saline. 
particularly alcoholic liver disease (ALD) and hepatitis C. ${ }^{11-16}$ High aPL titres are frequent in patients with alcoholic hepatitis or cirrhosis and the presence of aPL in these patients seems to reflect disease progression, showing significant correlation with disease severity. ${ }^{12}$ However, aPL can also be detected in alcoholics without liver damage. ${ }^{12}{ }^{17}{ }^{18}$ Furthermore, aPL are often associated with venous and arterial thrombosis ${ }^{4}$ : the presence of aPL in patients with chronic liver disease does not seem to be associated with thrombotic complications. ${ }^{16}$

Clinical and experimental evidence indicates that ethanol intoxication promotes free radical formation and oxidative liver injury. ${ }^{19}$ In particular, increased levels of lipid peroxidation products and protein carbonyls can be demonstrated in liver biopsies or in the sera obtained from patients with ALD. ${ }^{19-23}$ Furthermore, the formation of hydroxyethyl free radicals during ethanol metabolism can also be documented in patients with alcohol abuse. ${ }^{24}$

The aim of this work was to evaluate whether the development of aPL in patients with ALD might reflect ethanol mediated oxidative injury.

\section{Patients and methods}

PATIENTS AND CONTROL SUBJECT RECRUITMENT We enrolled 36 patients ( 27 men, nine women; mean age 51 years (range 31-73)) with alcoholic cirrhosis with or without hepatitis (ALD). The diagnosis was based on clinical, ecographycal, and laboratory criteria. Liver biopsy was available for 12 patients and showed the classical feature of micronodular cirrhosis. In three patients hepatocyte ballooning degeneration with Mallory's bodies and inflammatory infiltrates were also noted. Evaluation of the clinical severity of liver injury according to Maddrey's DF index or the Child Turcotte classification ${ }^{25}{ }^{26}$ revealed that $72 \%$ of patients in the ALD group had moderate or severe liver damage (DF values above 60) and 58\% belonged to Child group B or C. Fifteen heavy drinkers (11 men, four women; mean age 44 years (range 25-66)) with normal liver blood tests and no clinical or ecographycal evidence of liver disease were also investigated. All patients with alcohol abuse had an estimated mean daily ethanol intake over the previous 12 months above $100 \mathrm{~g}$ (table 1) and none had a previous history of co-exposure to drugs or toxins. Patients with alcohol abuse were negative for serum markers of hepatitis $B$ virus or for the presence of antibodies against hepatitis $\mathrm{C}$ virus (HCV), measured by a second generation enzyme linked immunoadsorbent assay (Abbott Laboratories, Chicago, Illinois, USA).

A group of 40 patients (29 men, 11 women; mean age 54 years (range 32-76)) suffering from $\mathrm{HCV}$ or hepatitis B virus chronic hepatitis or cirrhosis and without alcohol abuse were also investigated. In this group, $62 \%$ of patients were abstinent and the remaining had reported alcohol intake ranging from 10 to $50 \mathrm{~g}$ ethanol/ day. The severity of liver injury in these patients was comparable with that in patients with alcoholic liver damage. Aspartate aminotransferase, alanine aminotransferase, gamma glutamyltransferase, bilirubin, and prothrombin time were assayed in all patients using routine laboratory procedures. The biological characteristics of the patients studied are reported in table 1 .

Healthy controls were recruited among blood donors or university staff and consisted of 42 subjects ( 28 men, 14 women; mean age 47 years (range 33-66)). Alcohol intake among controls was below $20 \mathrm{~g}$ ethanol/day for women and was 40-60 g/day for men. At the time of blood testing, all subjects had abstained from alcohol for at least 48 hours. All subjects gave informed consent to the analysis and the study was planned according to the guidelines of the university ethics committee. Blood samples (5 $\mathrm{ml}$ ) were taken after an overnight fast and serum was used for ELISA tests.

ANTIPHOSPHOLIPID ANTIBODY DETERMINATION IgG reacting with $\beta_{2}$-GP1 and with cardiolipin were measured by commercial ELISA kits by, respectively, IMMCO Diagnostics Inc. (Buffalo, New York, USA) and IMTEC Immunodiagnostika GmbH (Berlin, Germany). Threshold values for anticardiolipin and anti- $\beta_{2}-\mathrm{GP} 1$ antibodies were determined previously in a representative sample of healthy controls from the same population.

\section{DETERMINATION OF IMMUNE REACTIVITY}

TOWARDS OXIDISED PHOSPHOLIPIDS

Phospholipid coated ELISA plates were prepared according to a standardised method proposed by Harris. ${ }^{1}$ Briefly, $30 \mu \mathrm{l}$ of cardiolipin solution in ethanol $(50 \mu \mathrm{g} / \mathrm{ml})$ were applied to each well and ethanol evaporated overnight at $4^{\circ} \mathrm{C}$ in air. After two washes with phosphate buffered saline (PBS), $0.3 \mathrm{ml}$ of coating buffer containing 3\% bovine serum albumin (BSA) in PBS, $\mathrm{pH} 7.4$, were added and the plates were further incubated for one hour at $37^{\circ} \mathrm{C}$ to block non-specific binding sites. The coated wells were washed three times with PBS. To avoid cardiolipin auto-oxidation in some preparations, $0.1 \mathrm{mmol} / 1$ diphenylphenylendiamine (DPPD) was added to cardiolipin ethanol solution and coating of the plates was carried out in sealed plastic bags filled with nitrogen. Cardiolipin coated plates were used immediately after preparation. For the assay, sera from patients and controls were diluted 1:50

Table 1 Biological characteristics of patients admitted to the study

\begin{tabular}{|c|c|c|c|c|c|c|c|}
\hline Group & $n$ & $\begin{array}{l}\text { Alcohol consumption } \\
\text { (g/day) }\end{array}$ & $A S T(U / l)$ & $A L T(U / l)$ & $G G T(U / l)$ & $\begin{array}{l}\text { Bilirubin } \\
\text { (ㅍmol/l) }\end{array}$ & $P T(\%)$ \\
\hline Alcoholic cirrhosis & 36 & $146(67)$ & $74(52)$ & $50(36)$ & $143(132)$ & $1.68(1.5)$ & $72(14)$ \\
\hline Non-alcoholic cirrhosis & 40 & $20(14)$ & $61(36)$ & $83(58)$ & $51(24)$ & $1.33(0.6)$ & $54(4)$ \\
\hline Heavy drinkers & 15 & $190(61)$ & $28(13)$ & $22(11)$ & $37(19)$ & $0.62(0.3)$ & $101(3)$ \\
\hline
\end{tabular}

AST, aspartate aminotransferase; ALT, alanine aminotransferase; GGT, gamma glutamyltransferase; PT, prothrombin time. Values are mean (SD). 
with the coating buffer and $0.2 \mathrm{ml}$ aliquots were added in duplicate to the appropriate wells and incubated for one hour at $37^{\circ} \mathrm{C}$. The plates were then washed three times with PBS $0.25 \%$ and further incubated with peroxidase linked goat antihuman IgG (dilution 1:6000) for 60 minutes at $37^{\circ} \mathrm{C}$. Antibody binding was revealed by addition of $0.15 \mathrm{ml}$ of a reaction mixture containing $0.4 \mathrm{mg} / \mathrm{ml}$ of L-phenylendiamine, $0.4 \mu \mathrm{l} / \mathrm{ml}$ hydrogen peroxide $(30 \%), 5.1 \mathrm{mg} / \mathrm{ml}$ citric acid, and 6.1 $\mathrm{mg} / \mathrm{ml}$ anhydrous $\mathrm{Na}_{2} \mathrm{HPO}_{4}, \mathrm{pH}$ 5.0. After 15 minutes the reaction was stopped by adding 50 $\mu \mathrm{l}$ of $2 \mathrm{~N} \mathrm{H}_{2} \mathrm{SO}_{4}$ and absorbance was measured at $490 \mathrm{~nm}$ using a Bio-Rad microplate reader (Bio-Rad Laboratories Inc., Hercules, California, USA). The average optical densities (od) in duplicated wells were subtracted from non-specific background for each serum sample obtained by performing the test in plates coated with ethanol but lacking the phospholipid.

\section{ASSESSMENT OF PHOSPHOLIPID OXIDATION}

The occurrence of phospholipid peroxidation under the conditions used for ELISA plate coating was monitored by measuring conjugated diene absorbance. Briefly, $0.5 \mathrm{mg} / \mathrm{ml}$ of cardiolipin solution were placed in glass test tubes and allowed to evaporate from the solvent by overnight incubation at $4^{\circ} \mathrm{C}$ in air. Cardiolipin was then resolubilised in $2 \mathrm{ml}$ hexane and conjugated diene absorbance was immediately measured at $234 \mathrm{~nm}$ using a Beckman DU650 UV visible spectrophotometer. Freshly prepared cardiolipin solution in hexane was used as reference.

PREPARATION OF PROTEIN ADDUCTS WITH LIPID PEROXIDATION PRODUCTS

Adducts between human serum albumin (HSA) and malondialdehyde (MDA) were prepared by reacting for two hours at $37^{\circ} \mathrm{C}$, $1 \mathrm{mg} / \mathrm{ml} \mathrm{HSA}$ with $50 \mathrm{mmol} / 1 \mathrm{MDA}$, obtained by acid hydrolysis of malondialdehyde-bisdimethylacetal. Unbound aldehyde was removed by overnight dialysis at $4^{\circ} \mathrm{C}$ against PBS, $\mathrm{pH}$ 7.4. The presence of MDA adducts was evaluated by measuring fluorescence intensity at $399 / 471 \mathrm{~nm}$ exc/em wavelength pairs according to Cominacini and colleagues. ${ }^{27}$ HSA complexed with reactive products of fatty acid oxidation was generated by thermal auto-oxidation of arachidonic acid (HSA-APP) and linoleic acid (HSA-LPP) according to Palinski and colleagues. ${ }^{28}$ Briefly, $10 \mathrm{mg}$ arachidonic acid or linoleic acid were transferred in a glass vial open to air and kept at $37^{\circ} \mathrm{C}$ for 72 hours. The yellow-brown reaction products were dissolved in $50 \mu \mathrm{l}$ methanol and suspended by vortexing in $1 \mathrm{ml}$ PBS containing $10 \mu \mathrm{M}$ EDTA, $\mathrm{pH}$ 7.4. Aliquots containing $3 \mathrm{mg}$ of oxidised fatty acids were added to $1 \mathrm{mg}$ HSA and incubated overnight at $20^{\circ} \mathrm{C}$. Modified HSA was dialysed overnight at $4^{\circ} \mathrm{C}$ against PBS, pH 7.4.
MEASUREMENT OF ANTIBODIES AGAINST LIPID PEROXIDATION DERIVED ANTIGENS

Polystyrene microwell plates for ELISA (Immunolon IV; Nunc, Fisher Scientific, St Louis, Missouri, USA) were coated for four hours at $37^{\circ} \mathrm{C}$ with $0.05 \mathrm{mg} / \mathrm{ml}$ of HSA conjugated with either MDA, arachidonic acid oxidation products (HSA-APP), or with the unmodified protein solubilised in $0.1 \mathrm{M}$ bicarbonate buffer, $\mathrm{pH}$ 9.6. After incubation, solutions were removed and replaced by $0.3 \mathrm{ml}$ of coating buffer containing 3\% BSA in PBS, pH 7.4. The plates were further incubated for one hour at $37^{\circ} \mathrm{C}$ to block non-specific binding sites. The coated wells were washed three times with PBS containing $0.25 \%$ Triton X-100. The sera of patients were diluted 1:50 with the coating buffer and added in duplicate as aliquots of $0.20 \mathrm{ml}$ to the appropriate wells and incubated for one hour at $37^{\circ} \mathrm{C}$. After washing three times with PBS- $0.25 \%$ Triton X-100, IgG binding was revealed by peroxidase linked goat antihuman IgG as described above. The results were expressed by subtracting the spectrophotometric readings of each serum sample in the wells containing the unmodified HSA.

IMMUNOCOMPETITION EXPERIMENTS

Sera of ALD patients positive for anti- $\beta_{2}-\mathrm{GP} 1$ antibodies were diluted 1:50 with coating buffer and $300 \mu$ laliquots placed in each well of microtitre plates coated with $\beta_{2}$-GP1 (IMTEC Immunodiagnostika $\mathrm{GmbH}$, Berlin, Germany) or HSA. After overnight incubation at $4^{\circ} \mathrm{C}, 150$ $\mu \mathrm{l}$ aliquots were added in duplicate to ELISA plates coated with oxidised cardiolipin, HSAMDA, HSA-APP, or HSA-LPP, and antibody binding was assayed as described above.

DATA ANALYSIS AND STATISTICAL CALCULATIONS Data are expressed as mean (SD). Statistical analysis was performed by Instat-3 statistical software (GraphPad Software Inc, San Diego, California, USA) using one way ANOVA with Bonferroni's correction for multiple comparisons when more than two groups were analysed. Pearson's $r$ values were used for estimation of correlation. Distribution normality of all groups was preliminary verified by the Komolgorov-Smirnov test. Significance was taken at the $5 \%$ level.

\section{MATERIALS}

Arachidonic acid, linoleic acid, L-phenylendiamine, cardiolipin, and fatty acid free human serum albumin (fraction V) were from Sigma Chemical Co. (St Louis, Missouri, USA). DPPD and malondialdehyde-bis-dimethylacetal were supplied by Fluka Chemie AG (Buchs, Switzerland).

\section{Results}

The use of commercial kits for the aPL assay based on binding to cardiolipin coated ELISA plates revealed that 20 of $36(55 \%)$ patients with alcoholic cirrhosis with or without alcoholic hepatitis (ALD) had anticardiolipin IgG levels above the threshold value (15 GPL units $/ \mathrm{ml}$ ) while one healthy control was positive for anticardiolipin IgG. Mean IgG reactivity 
towards cardiolipin among ALD patients was 22.7 (14) U/ml (range 11.8-71.1), significantly higher $(\mathrm{p}<0.001)$ than that in control subjects (11.8 (1.3) U/ml; range 10.9-17.6).

It has been shown that the unsaturated fatty acid component of cardiolipin rapidly autooxidises when exposed to air. ${ }^{9}$ Determination of conjugated diene absorbance at $234 \mathrm{~nm}$, as an index of lipid peroxidation, revealed that overnight incubation at $4^{\circ} \mathrm{C}$ generally used for coating ELISA plates with phospholipids, ${ }^{1}$ appreciably increased the absorbance $\left(\operatorname{od}_{234} \mathrm{~nm}\right.$ 0.276) of cardiolipin samples compared with freshly prepared solutions ( $\left.\operatorname{od}_{234 \mathrm{~nm}} 0.110\right)$. Cardiolipin auto-oxidation was however completely prevented $\left(\mathrm{od}_{234 \mathrm{~nm}} 0.112\right)$ by incubation under a nitrogen atmosphere in the presence of the antioxidant DPPD $100 \mu \mathrm{mol} / \mathrm{l}$. When the antiphospholipid reactivity of sera was retested using ELISA plates coated with either oxidation protected cardiolipin or cardiolipin allowed to auto-oxidise in air, a dramatic difference was observed. As shown in fig 1, binding of IgG from ALD sera to oxidation protected cardiolipin $\left(\mathrm{od}_{490 \mathrm{~nm}} 0.274(0.184)\right)$ was significantly lower $(\mathrm{p}<0.0001)$ than that to oxidised cardiolipin $\left(\mathrm{od}_{490 \mathrm{~nm}} 0.491(0.340)\right)$. Reactivity of control sera with either form of cardiolipin was unchanged $\left(\operatorname{od}_{490} \mathrm{~nm} 0.205\right.$ $(0.103) v 0.187$ (0.111)) (fig 1). When tested with oxidised cardiolipin, 21 of 36 (58\%) ALD sera showed IgG binding above the threshold value in controls given by mean od ${ }_{490 \mathrm{~nm}}$ values +2 SD (0.408). However, only eight of 36 (19\%) ALD sera still displayed increased reactivity when tested with oxidation protected cardiolipin (cut off value 0.387) (fig 1). Furthermore, reactivity of ALD and control sera to oxidation protected cardiolipin was not statistically different. This was not due to interference of DPPD with the antibody reaction as addition of the antioxidant to already oxidised cardiolipin preparations did not affect IgG binding (not shown). No

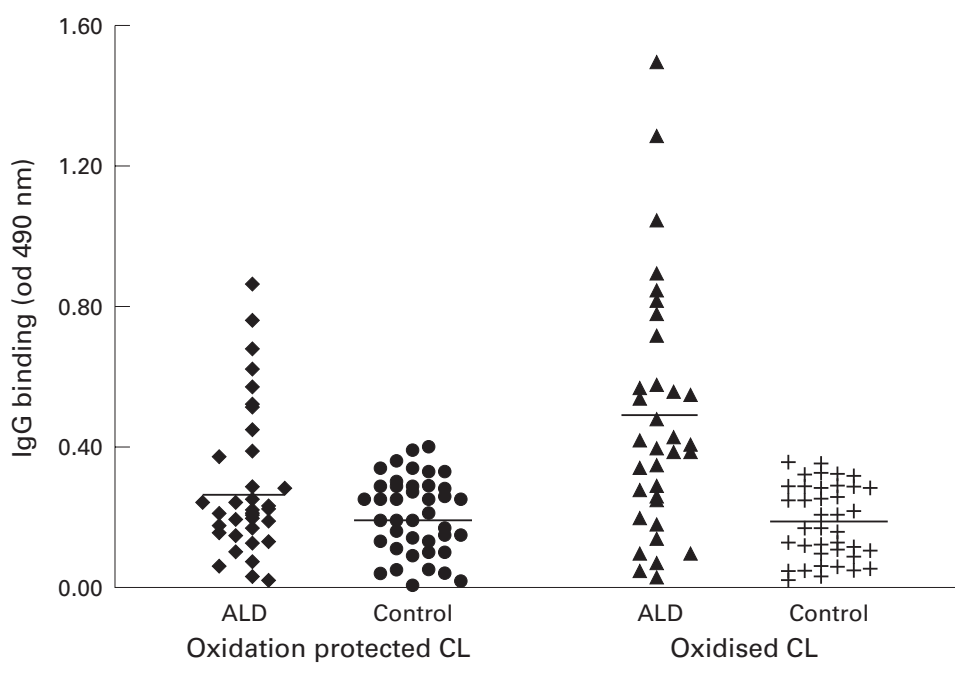

Figure 1 Immune reactivity against native or oxidised cardiolipin (CL) of sera from 36 patients with alcoholic cirrhosis with or without alcoholic hepatitis (ALD) and from 42 healthy control subjects. IgG binding to ELISA plates coated with CL allowed to auto-oxidise in air or oxidation protected CL was evaluated, as reported in the patients and methods section. The horizontal bars represent median values in each group. Values in each group were normally distributed, as evaluated by the Komolgorov-Smirnov test. increase in reactivity towards oxidised cardiolipin was apparent in a group of 15 heavy drinkers without clinical or biochemical evidence of liver damage $(0.153(0.09))$ or in 40 patients with cirrhosis unrelated to alcohol abuse $(0.284(0.10))$.

Lipid hydroperoxides and aldehyde generated during lipid peroxidation are known to interact with proteins forming adducts that are immunogenic. ${ }^{28}$ We observed that ALD patients had enhanced level of circulating IgG against HSA modified by the reaction with malondialdehyde (HSA-MDA) or with oxidation products derived from arachidonic acid (HSA-APP) $\left(\operatorname{od}_{490} \mathrm{~nm} 0.79(0.27)\right.$ and 0.47 (0.25), respectively, $v 0.45(0.14)$ and 0.11 $(0.08)$ in healthy controls; $\mathrm{p}<0.001)$. Individual levels of antibodies against oxidised cardiolipin were correlated with those of IgG directed against HSA-APP or HSA-MDA $(r=0.67$ and 0.60 , respectively; $\mathrm{p}<0.0001)$. Moreover, among ALD patients, levels of antibodies against lipid peroxidation products were significantly higher in subjects positive for antioxidised cardiolipin antibodies than in the negative group (fig 2), indicating a relationship between the presence of an immune response against phospholipids and ethanol induced oxidative injury. On the other hand, titres of IgG against HSA-MDA and HSA-APP were not significantly increased in heavy drinkers without liver damage $\left(\operatorname{od}_{490 \mathrm{~nm}} 0.450(0.140)\right.$ and 0.039 (0.030), respectively) or in nonalcoholic cirrhotics $\left(\operatorname{od}_{490 \mathrm{~nm}} 0.590(0.220)\right.$ and 0.196 (0.151), respectively).

Antiphospholipid antibodies can also be detected by their ability to recognise complexes formed by $\beta_{2}$-GP1 and phospholipids. ${ }^{4}$ To avoid the confounding factor associated with cardiolipin oxidation state, antiphospholipid reactivity associated with ALD was reevaluated by assaying anti- $\beta_{2}$-GP1 antibodies. IgG reactivity towards $\beta_{2}$-GP1 (46.1 (29.5) $\mathrm{U} / \mathrm{ml}$ (range 13-150)) was significantly higher $(\mathrm{p}<0.001)$ in ALD patients than in control

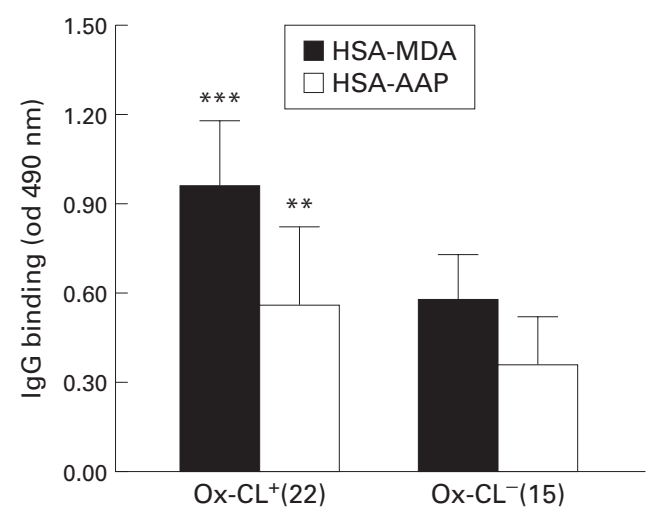

Figure 2 Reactivity with antigens derived from the reaction of proteins with lipid peroxidation products of sera from patients with alcoholic liver disease positive $\left(O x-C L^{+}\right)$ or negative $\left(\mathrm{Ox}-\mathrm{CL}^{-}\right)$for the presence of antibodies against oxidised cardiolipin. Human serum albumin (HSA) modified by the reaction with either malondialdehyde (HSA-MDA) or products from arachidonic acid peroxidation (HSA-APP) were used as antigens, as reported in the patients and methods section. Results are mean (SD) ELISA values. ${ }^{\star \star} p<0.01,{ }^{\star}{ }^{\star *} p<0.0001$ versus $\mathrm{Ox}-\mathrm{CL}^{-}$subjects. 


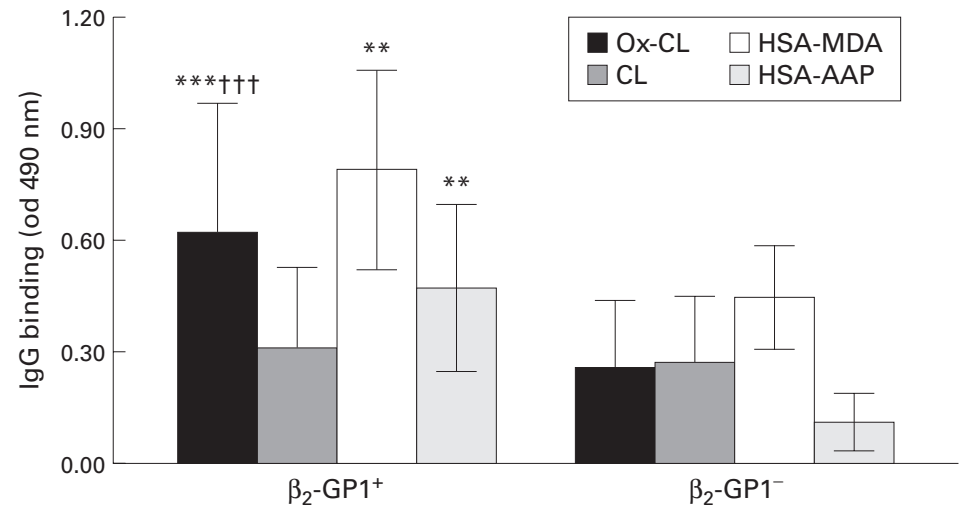

Figure 3 Reactivity with oxidised cardiolipin or with antigens derived from the reaction of proteins with lipid peroxidation products of sera from patients with alcoholic liver disease positive $\left(\beta_{2}-G P 1^{+} ; n=23\right)$ or negative $\left(\beta_{2}-G P 1^{1} ; n=13\right)$ for the presence of antibodies against $\beta_{2}$-glycoprotein 1. ELISA plates coated with oxidation protected $(C L)$ or oxidised $(O x-C L)$ cardiolipin, or with malondialdehyde (HSA-MDA) or products from arachidonic acid peroxidation (HSA-APP) were used to evaluate IgG binding. Results are mean (SD) ELISA values. ${ }^{\star *} p<0.005,{ }^{\star *} p p<0.001$ versus $\beta_{2}-$ GP1 negative subjects; $t+t p<0.001$ versus native cardiolipin.

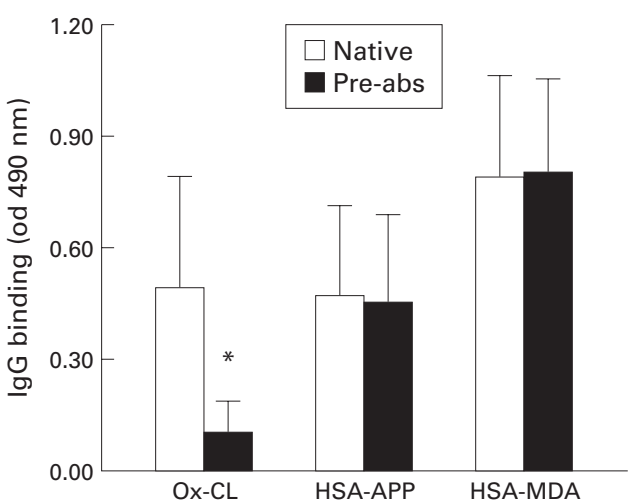

Figure 4 Reactivity with oxidised cardiolipin or human serum albumin (HSA) complexed with lipid peroxidation products following preabsorption with the $\beta_{2}$ glycoprotein 1 $\left(\beta_{2}-G P 1\right)$ of sera from alcoholic patients. Fifteen sera from patients with alcoholic liver disease displaying high titres of Ig $G$ against both cardiolipin and $\beta_{2}-G P 1$ were incubated overnight at $4^{\circ} \mathrm{C}$ on irradiated ELISA plates coated with $\beta_{2}-G P 1$ and tested for IgG binding to oxidised cardiolipin $(O x-C L)$ or HSA modified by reaction with malondialdehyde (HSA-MDA) or products of arachidonic acid peroxidation (HSA-APP). Results are mean (SD)

ELISA values obtained with native sera or $\beta_{2}-G P 1$

preabsorbed sera. ${ }^{\star} p<0.0001$ versus non-pre-absorbed sera. anti- $\beta_{2}-\mathrm{GP} 1$ IgG. $\beta_{2}-\mathrm{GP} 1$ positive ALD sera showed titres of IgG recognising oxidised cardiolipin significantly higher $(\mathrm{p}<0.001)$ than $\beta_{2}$-GP1 negative sera (fig 3 ), despite the fact that total circulating IgG levels were not significantly different between the two groups (15.89 (7.37) g/l v 13.26 (3.01) g/l). Furthermore, a strong positive correlation $(r=0.85$; $\mathrm{p}<0.0001)$ was evident when individual values of anti- $\beta_{2}-\mathrm{GP} 1 \mathrm{IgG}$ were compared with those of antibodies directed against oxidised cardiolipin. Thus we postulated that aPL detected in ALD sera might recognise oxidatively modified cardiolipin complexed with $\beta_{2}$-GP1. Indeed, preadsorption of ALD sera displaying both anticardiolipin and anti- $\beta_{2}-\mathrm{GP} 1$ reactivity on ELISA plates coated with $\beta_{2}-\mathrm{GP} 1$ reduced IgG binding to oxidised cardiolipin by about $80 \%$ (fig 4). ALD patients positive for $\beta_{2}-\mathrm{GP} 1$ also showed reactivity towards HSA-MDA and HSA-AAP significantly higher $(\mathrm{p}<0.005)$ than that of $\beta_{2}$-GP1 negative subjects (fig 3 ). To exclude the possibility that antibodies directed towards MDA or APP derived epitopes might account for antiphospholipid reactivity by recognising complexes between $\beta_{2}$-GP1 and products of phospholipid oxidation, further experiments were performed. As shown in fig 4, preadsorption on ELISA plates coated with $\beta_{2}$-GP1 that almost abolished recognition of oxidised cardiolipin by aPL positive sera did not affect IgG binding to HSA-APP or HSA-MDA. Similarly, preadsorption of ALD sera on plates coated with oxidised cardiolipin also did not interfere with the recognition of antigens formed by the reaction of HSA with oxidation products originating from autooxidation of linoleic acid (HSA-LPP), the main unsaturated fatty acid of cardiolipin $\left(\operatorname{od}_{490} \mathrm{~nm}\right.$ $0.280(0.09)$ in preadsorbed sera $v \operatorname{od}_{490} \mathrm{~nm}$ $0.272(0.120)$ in non-preadsorbed sera). However, in the same experiments, preadsorption with HSA-LPP decreased $\left(\operatorname{od}_{490}{ }_{\mathrm{nm}} 0.049\right.$ (0.07)) binding of ALD sera to the same antigen by $82 \%$. Therefore, antibodies towards antigens derived from lipid peroxidation products did not account for the reactivity against oxidised phospholipids detected in the sera of ALD patients.

Previous studies have shown that antiphospholipid immune reactivity was prevalent in patients with alcoholic hepatitis or cirrho$\operatorname{sis}^{11} 1214$ and was particularly higher in nonabstaining subjects or in cirrhotics with severe liver damage (Child grades B and C) ${ }^{14}$ We have observed that individual reactivity against oxidised cardiolipin was positively correlated $(r=0.65 ; \mathrm{p}<0.0001)$ with Maddrey's DF values. Furthermore, circulating levels of IgG against oxidised cardiolipin were significantly $(\mathrm{p}<0.0002)$ higher in ALD patients with severe liver injury (Maddrey's DF index >90) compared with patients with moderate or mild liver damage (Maddrey's DF index <90) (fig 5). A statistically significant difference $(\mathrm{p}<0.01)$ was also observed when the same patients were subgrouped according to the Child-Turcotte classification (fig 5). This indicated that the pholipids might actually account for high aPL titres observed in association with severe ALD.

\section{Discussion}

In recent years several reports have highlighted the association between alcoholic intoxication and the presence of circulating antiphospholipid antibodies (aPL). ${ }^{11} 121718$ In these studies, the prevalence of aPL among patients with ALD ranged from $48 \%$ up to $81 \%$. These values are in agreement with the prevalence of aPL (about 55\%) observed in our group of patients with alcoholic liver cirrhosis. However, aPL have also been found in $31 \%$ of alcoholics with abnormal liver function but without cirrhosis $^{12}$ and in $15-17 \%$ of alcoholics without clinical or biochemical signs of liver injury. ${ }^{17} 18$ Anticardiolipin antibodies can also be detected in patients with $\mathrm{HCV}$ and are more frequent in subjects with advanced cirrhosis. ${ }^{13-15}$ None the presence of antibodies against oxidised phos- 

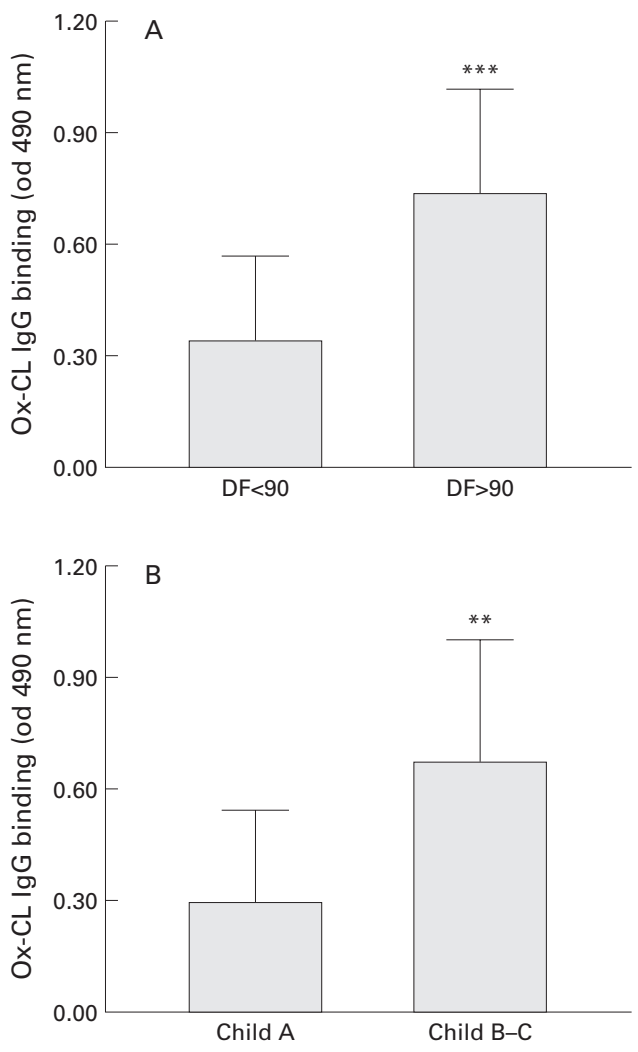

Figure 5 Reactivity against oxidised cardiolipin $(\mathrm{Ox}-\mathrm{CL})$ in relation to the severity of alcohol liver injury. The clinical severity of hepatic damage was estimated according to Maddrey's DF classification ${ }^{23}$ (A) or Child-Turcotte classification (B). Patients with DF values less than 90 $(n=26)$ were considered to have mild or moderate hepatic injury and patients with $D F$ values greater than 90 $(n=10)$ represented the group with severe damage. Child $A$ group consisted of 15 patients, while 21 subjects belonged to Child B-C groups. The optical density (od) values in each group were normally distributed, as evaluated by the Komolgorov-Smirnov test. ${ }^{\star \star \star} p<0.0002$ versus $D F<90$; ${ }^{\star *} p<0.01$ versus Child $A$ group.

less, the prevalence of anticardiolipin antibodies associated with $\mathrm{HCV}$ infection is much lower than in patients with ALD. ${ }^{14}{ }^{15}$ Coexistence of $\mathrm{HCV}$ infection with alcohol abuse was taken into consideration in the present study as well as in previous reports, by excluding patients with evidence of HCV infection.

Little is known about the nature of the antigens recognised by aPL associated with ALD. These antibodies have been detected by binding to cardiolipin immobilised on the surface of ELISA plates, but immune reactivity with other classes of phospholipids (that is, phosphatidylcholine, phosphatidylethanolamine, phosphatidylserine, phosphatidylglycerol) have also been reported. ${ }^{12}$ An elegant study by Hörkkö et al demonstrated that selected sera from women with antiphospholipid syndrome bind to neo-epitopes generated when cardiolipin undergoes oxidation. ${ }^{9}$ The fatty acid content of cardiolipin largely consists (about $90 \%$ ) of linoleic acid, and peroxidation of these fatty acids readily occurs when microtitre ELISA plates are exposed to air during the coating procedure. ${ }^{9}$ We now report that anticardiolipin antibodies detected in sera of patients with ALD weakly react with native cardiolipin when it is protected from spontaneous auto-oxidation, but recognise instead antigens present in oxidised cardiolipin.

Several lines of evidence indicate the involvement of oxidative damage in alcoholic liver damage. Liver biopsies or sera from patients with ALD contain higher amounts of lipid peroxidation products (conjugated dienes, malondialdehyde, 4-hydroxynonenal, $\mathrm{F}_{2}-$ isoprostanes) and protein carbonyls than similar specimens from non-drinking subjects or patients with liver diseases unrelated to alcohol. ${ }^{19-23}$ By reacting with proteins, free radicals, lipid hydroperoxides, and aldehydes generated from lipid peroxidation can produce a variety of adducts most of which are antigenic and can stimulate the production of specific antibodies. ${ }^{28}{ }^{29}$ Increased levels of antibodies against hydroxyethyl free radicals and MDAprotein adducts have previously been detected in patients with ALD. ${ }^{24}{ }^{30-32}$ We have observed that individual levels of IgG directed against adducts between serum albumin and either malonildialdehyde (HSA-MDA) or arachidonic acid oxidation (HSA-APP) products are closely associated with those of antioxidised cardiolipin antibodies. Furthermore, among patients with ALD, levels of antibodies against lipid peroxidation products are significantly higher in groups positive for antioxidised cardiolipin antibodies than in negative subjects. Conversely, titres of IgG antioxidised cardiolipin in heavy drinkers without liver damage or in non-alcoholic cirrhotics that do not display evidence of oxidative injury are not significantly different from those in healthy controls. Thus we propose that the development of antiphospholipid autoimmunity associated with ALD might be related to the pro-oxidant conditions induced by ethanol. Such a hypothesis is consistent with experimental observations suggesting that oxidative modifications of phospholipids triggers the development of the antiphospholipid immune response. ${ }^{1033}$ Furthermore, Iuliano et al have shown that among patients affected by systemic lupus erythematosus, those positive for aPL have higher urinary excretion of the lipid peroxidation product $\mathrm{F}_{2}$-isoprostane than similar patients not displaying aPL reactivity. ${ }^{34}$

It is now largely accepted that the plasma protein $\beta_{2}-\mathrm{GP} 1$ or apolipoprotein $\mathrm{H}$ plays a key role in aPL reactivity, serving as a cofactor for antibody binding to phospholipids. ${ }^{34}$ In the liquid phase, $\beta_{2}-\mathrm{GP} 1$ is not recognised by aPL but conformational changes occurring following interaction with phospholipids render $\beta_{2}$-GP1 antigenic for aPL. ${ }^{7}$ We have observed that a high proportion (64\%) of sera from patients with ALD displayed reactivity with $\beta_{2}$-GP1. A close correlation $\quad(r=0.85$; $\mathrm{p}<0.0001)$ was also seen between individual levels of anti- $\beta_{2}-\mathrm{GP} 1$ IgG and those of antibodies directed against oxidised cardiolipin. Interestingly, only $\beta_{2}$-GP1 positive sera recognised antigens in oxidised cardiolipin and preadsorption of $\beta_{2}$-GP1 positive sera with $\beta_{2}$-GP1 attached to ELISA microtitre plates decreased antibody binding to oxidised cardiolipin antigens by $80 \%$. This indicates that aPL 
associated with alcoholic damage are directed against epitopes formed by the interaction of $\beta_{2}$-GP1 with oxidised phospholipids. Hörkkö et al have suggested that the neo-epitopes recognised by some aPL might consist of adducts formed between breakdown products of oxidised phospholipids and $\beta_{2}-\mathrm{GP} 1 .^{9}{ }^{10}$ As mentioned above, circulating levels of antibodies directed towards lipid peroxidation related epitopes are increased in ALD patients. Furthermore, ALD patients displaying $\beta_{2}$-GP1 reactivity have higher serum levels of IgG recognising HSA-MDA or HSA-APP than $\beta_{2}$-GP1 negative subjects. However, preadsorption of ALD sera with $\beta_{2}-\mathrm{GP} 1$ that almost abolishes antibody binding to oxidised cardiolipin does not interfere with binding to epitopes in HSA complexed with MDA or with oxidised arachidonic or linoleic acids. We conclude that antibodies directed against protein adducts with lipid peroxidation products, such as MDA or lipid hydroperoxides, do not account for the immune reactivity towards oxidised cardiolipin. We propose that antiphospholipid antibodies detected in patients with ALD rather recognise complexes between $\beta_{2}-\mathrm{GP} 1$ and oxidised cardiolipin.

Few data are available concerning the possible role of aPL in alcohol mediated liver injury. Antiphospholipid immune reactivity can be detected in patients with alcoholic hepatitis or cirrhosis $^{11214}$ and particularly in nonabstaining patients and in cirrhotics with severe liver damage (Child grades B and C).$^{14}$ We have detected higher values of IgG against oxidised cardiolipin in ALD patients with severe liver damage. Moreover, a positive correlation $(0.65 ; \mathrm{p}<0.0001)$ was evident between individual antioxidised cardiolipin IgG and Maddrey's DF values. This indicates that the high prevalence of aPL in association with severe ALD can be ascribed to the presence of antibodies targeting oxidised phospholipids. The clinical significance of aPL associated with alcoholic liver damage is at the moment unknown. The presence of aPL in patients with liver disease is not associated with an increased risk of thrombotic complications. ${ }^{16}$ Recent studies have proposed a possible link between aPL and cell death by apoptosis by showing that mice injected intravenously with syngenic apoptotic thymocytes develop anticardiolipin antibodies. ${ }^{35}$ Consistently, sera of patients with antiphospholipid syndrome bind specifically to apoptotic thymocytes but not to viable cells by recognising epitopes generated by the interaction of $\beta_{2}$-GP1 with plasma membrane cardiolipin. ${ }^{36}$ Interestingly, plasma membrane antigens in apoptotic cells are also targets for monoclonal antibodies recognising oxidised phospholipids. ${ }^{38}$ Alcoholic liver injury is associated with stimulation of hepatocyte apoptosis ${ }^{39}$ and oxidative events have been proposed to play a role in triggering apoptotic changes in hepatocytes exposed to ethanol. ${ }^{40}$ Thus it is possible that oxidised phospholipids in the membranes of apoptotic hepatocytes might act as a stimulus as well as a target for aPL detected in ALD patients.
In conclusion, our results indicate that aPL detected in patients with ALD have a strict relationship with ethanol induced oxidative damage and preferentially target oxidised phospholipids complexed with $\beta_{2}$-GP1. These findings suggest a possible role for oxidative mechanisms in the development of aPL associated with alcoholic liver injury.

This work was supported by grants from the Italian Ministry for University and Scientific and Technological Research (Research Program: Redox Regulation of Cellular Processes) and by the National Research Council (contribution No 96.00073.CT04).

1 Harris NE. Antiphospholipid antibodies. Br f Haematol 1990;74:1-9.

2 Mc Neil HP, Chesterman CN, Krilis SA. Immunological and clinical importance of antiphospholipid antibodies. Adv Immunol 1991;49:193-280.

3 Hughes GRV. The antiphospholipid syndrome: ten years on. Lancet 1993;342:341-4.

4 Greaves M. Antiphospholipid antibodies and thrombosis. Lancet 1999;352:1384-53.

5 McNeil HP, Simpson J, Chesterman CN, et al. Antiphospholipid antibodies are directed against a complex antigen that include a lipid binding inhibitor of coagulation $\beta_{2}$-glycoprotein 1 (apolipoprotein H). Proc Natl Acad Sci USA 1990;87:4120-4

6 Galli M, Comfurius P, Maassen C, et al. Anticardiolipin antibodies directed not to cardiolipin but to a plasma cofactor. Lancet 1990;355:1544-7.

7 Chamley LW, Duncalf AM, Konarkowska B, et al. Conformational altered $\beta_{2}$-glycoprotein 1 is the antigen of anti-cardiolipin autoantibodies. Clin Exp Immunol 1999; 115:571-6.

8 Matsuura E, Igarashi Y, Yasuda T, et al. Anticardiolipin antibodies recognise $\beta_{2}$-glycoprotein 1 structure altered by oxygen modified solid phase surfaces. F Exp Med 1994; 179:457-62.

9 Hörkkö S, Miller E, Dudl E, et al. Antiphospholipid antibodies are directed against epitopes of oxidized phospholipids. Recognition of cardiolipin by monoclonal antibodies to epitopes of oxidized low density lipoprotein. $\mathcal{F}$ Clin Invest 1996;98:815-25.

10 Hörkkö S, Miller E, Branch DW, et al. The epitopes for some antiphospholipid antibodies are adducts of oxidized phospholipid and $\beta_{2}$-glycoprotein 1 (and other proteins). Proc Natl Acad Sci USA 1997;94:10356-61.

11 Bird G, Millis P, Smith D, et al. Antibodies to phospholipid in alcoholic liver disease. BMf 1994;309:1161.

12 Chedid A, Chadalawada KR, Morgan TR, et al. Phospholipid antibodies in alcoholic liver disease. Hepatol 1994;20: 1465-71.

13 Prieto J, Yuste JR, Beloqui O, et al. Anticardiolipin antibodies in chronic hepatitis C. Implication of hepatitis $\mathrm{C}$ virus as the cause of the antiphospholipid syndrome. Hepatology 1996;23:204-11.

14 Biron C, Andreani H, Blanc P, et al. Prevalence of antiphospholipid antibodies in patients with chronic liver disease related to alcohol or hepatitis $\mathrm{C}$ virus: correlation with liver injury. F Lab Clin Med 1998;131:243-50.

15 Leroy V, Arvieux J, Jacob MC, et al. Prevalence of anticardiolipin, anti- $\beta_{2}$-glycoprotein 1 and anti-prothrombin antibodlipin, anti- $\beta_{2}$-glycoprotein 1 and anti-prothrombin antibod-
ies in chronic hepatitis C. Br f Heamatol 1998;101:468-74.

16 Maggia A, Maraglione M, Cascavilla I, et al. Anticardiolipin antibodies in patients with liver disease. Am $\mathcal{F}$ Gastroenterol 1999;94:2983-7.

17 Biron C, Lalloyer N, Tonnelot JL, et al. Anticardiolipin antibodies and acute alcoholic intoxication. Lupus 1995;4:48690.

18 Zima T, Fialova L, Mikulikova L, et al. Antibodies against phospholipids and oxidized LDL in alcoholic patients. Physiol Res 1998;47:351-5.

19 Nordmann R, Ribière C, Rouach H. Implication of free radical mechanisms in ethanol induced cellular injury. Free Radic Biol Med 1992;12:219-40.

20 Clot $\mathrm{P}$, Tabone M, Aricò S, et al. Monitoring oxidative damage in patients with liver cirrhosis and different daily alcoage in patients with liver cirrhosis
hol intake. Gut 1994;35:1637-43.

21 Grattagliano I, Vendemiale G, Sabbà G, et al. Oxidation of circulating proteins in alcoholics: role of acetaldehyde and xanthine oxidase. F Hepatol 1996;25:28-36.

22 Aleynik SI, Leo MA, Aleynik MK, et al. Increased circulating products of lipid peroxidation in patients with alcoholic liver disease. Alcohol Clin Exp Res 1998;22:192-6.

23 Hill DB, Awad JA. Increased urinary $\mathrm{F}_{2}$-isoprostane excretion in alcoholic liver disease. Free Radic Biol Med 1999;26:656-60.

24 Clot P, Bellomo G, Tabone M, et al. Detection of antibodies against proteins modified by hydroxyethyl free radicals in patients with alcoholic cirrhosis. Gastroenterology 1995;108: 201-7.

25 Maddrey WC, Boitnott JK, Bedine MS, et al. Corticosteroid therapy of alcoholic hepatitis. Gastroentology 1978;75:193-9. 26 Conn HO. A peek at the Child-Turcotte classification. Conn HO. A peek at the
Hepatology 1981;1:673-6. 
27 Cominacini M, Garbin U, Davoli A, et al. A simple test for predisposition to LDL oxidation based on the fluorescence development during copper-catalyse
tion. F Lipid Res 1991;32:349-58.

28 Palinski W, Hörkkö S, Miller E, et al. Cloning of monoclonal autoantibodies to epitopes of oxidized lipoproteins from apolipoprotein E-deficient mice. Demonstration of epitopes of oxidized low density lipoproteins in human plasma. $\mathcal{F}$ Clin Invest 1996;98:800-14.

29 Palinski W, Ylä-Herttuala S, Rosenfeld ME, et al. Antisera and monoclonal antibodies specific for epitopes generated during the oxidative modification of low density lipoprotein. Atherosclerosis 1990;10:325-35.

30 Lecomte E, Herberth B, Pirrolet P, et al. Effect of alcohol consumption on blood antioxidant nutrients and oxidative stress indicators. Am 7 Clin Nutr 1994;60:255-61.

31 Dupont I, Lucas D, Clot P, et al. Cytochrome P4502E1 inducibility and hydroxyethyl radical formation among inducibility and hydroxyethyl radical

32 Rolla R, Vay D, Mottaran E, et al. Detection of circulating antibodies against malondialdehyde-acetaldehyde adducts in patients with alcoholic liver disease. Hepatology 2000;31: in patien

33 Itabe H, Yamamoto H, Suzuki M, et al. Oxidized phosphatidylcholine that modify proteins. Analysis by monoclonal antibodies against oxidized low density lipoprotein. $f \mathrm{Biol}$ Chem 1996;271:33208-17.
34 Iuliano L, Praticò D, Ferro D, et al. Enhanced lipid peroxidation in patients positive for antiphospholipid antibodies. Blood 1997;90:3931-5.

35 Mevorach D, Zhou JL, Song X, et al. Systemic exposure to irradiated cells induces autoantibody production. 7 Exp Med 1998; 188:387-92.

36 Prince BE, Rauch J, Shia MA, et al. Anti-phospholipid autoantibodies bind to apoptotic, but not viable thymocytes in a $\beta_{2}$-glycoprotein 1-dependent manner. F Immunol 1996; 157:2201-8.

37 Sorice M, Circella A, Misasi R, et al. Cardiolipin on the surface of apoptotic cells as a possible trigger for antiphospholipid antibodies. Clin Exp Immunol 2000;122:27784 .

38 Chang MK, Bergmark C, Laurila A, et al. Monoclonal antibodies against oxidized low-density lipoprotein bind to apoptotic cells and inhibit their phagocytosis by elicited macrophages: Evidence that oxidation-specific epitopes mediate macrophage recognition. Proc Natl Acad Sci USA 1999;96:6353-8.

39 Nanji AA. Apoptosis and alcoholic liver disease. Semin Liver Dis 1998;18:187-90.

40 Kurose I, Higuchi H, Miura S, et al. Oxidative stressmediated apoptosis of hepatocytes exposed to acute ethanol intoxication. Hepatology 1997; 25:368-78. 